\title{
Novel Passive Ceramic Based Semi-dry Electrodes for Recording Electroencephalography Signals from the Hairy Scalp
}

\author{
Guangli Li ${ }^{\text {a }}$, Dan Zhang ${ }^{\text {b }}$, Sizhe Wang ${ }^{\text {a }}$, Yanwen Y. Duan ${ }^{\text {a, * }}$ \\ ${ }^{a}$ College of Chemistry and Molecular Sciences, Wuhan University, Wuhan 430072, China \\ ${ }^{\mathrm{b}}$ Department of Psychology, School of Social Sciences, Tsinghua University, Beijing 100084, \\ China
}

*Correspondence to:

Dr. Y. Y. Duan

College of Chemistry and Molecular Sciences

Wuhan University

Wuhan Luojia Mountain, 430072

Wuhan, China

Tel.: +86-27-68752858

Fax: +86-27-68753383

Email: yduan@whu.edu.cn 


\begin{abstract}
This study reports on a novel passive ceramic-based semi-dry electrode prototype for electroencephalography (EEG) applications. With the help of capillary forces of the porous ceramics pillars, the semi-dry electrodes build a stable electrode/scalp interface by penetrating hair and releasing a small amount saline in a controlled and sustained manner. The semi-dry electrode/scalp impedances were low and stable $(44.4 \pm 16.9 \mathrm{k} \Omega, \mathrm{n}=10)$, and the variation between nine different positions was less $5 \mathrm{k} \Omega$. The semi-dry electrodes have shown nonpolarization characteristics and the maximum difference of equilibrium potential between eight electrodes was $579 \mu \mathrm{V}$. The semi-dry electrodes demonstrated long-term stability, and the impedance only increased by $20 \mathrm{k} \Omega$ within $8 \mathrm{~h}$. EEG signals were simultaneously recorded using a 9-channel gel-based electrode and semi-dry electrode arrays setup on ten subjects. The average temporal cross-correlation between them in the eyes open/closed and the steady state visually evoked potentials (SSVEPs) paradigm were $0.938 \pm 0.037$ and $0.937 \pm 0.027$ respectively. Spectral analyses revealed similar response patterns with expected functional responses. Together with the advantages of quick setup, self-application and cleanliness, the result suggests the semi-dry electrode is suitable for emerging real-world EEG applications, such as brain-computer interfaces and wearable EEGs.
\end{abstract}




\section{Introduction}

Electroencephalography (EEG) is nowadays the most widely used non-invasive brain imaging technique due to its excellent temporal resolution, high portability and relative low cost [1]. In recent years, the emerging EEG-based brain-computer interface (BCI) applications and wearable devices have attracted extensive attention from academic and industry circles. Compared to the requirements of electrode sensors used in traditional clinics and laboratories, more attention has been paid to friendly and convenient use in real-world application scenarios such as physiological monitoring [2-4], neuro-feedback training [5, 6] and neuro-marketing [7, 8], etc. However, despite all the recent technological advances in acquisition electronics and signal processing, convenient and reliable EEG electrodes still remain an important technological challenge.

Detecting high quality EEG signals depends on a reliable electrical path between electrode and scalp, which requires non-polarizable electrodes with low and stable electrode/scalp impedance. Neural signals are carried by ionic currents to the scalp surface via the body fluid, then recorded by the electrodes placed on the scalp. The electrode converts ionic current to electronic current, before sending the signals to an amplifier and subsequent signal processing. Here, conductive gel plays an important role of carrying bioelectrical signals (weak ionic current impulse) and builds an electronic/ionic interface at the electrode surface (referred to as the double layer). Conductive gels assist electrodes to form a stable electrode potential, minimizes electrode polarization and reduces electrode/scalp interface impedance.

So far, gel-based electrodes have become the main choice for recording EEG in clinics 
and research laboratories due to its excellent signal to noise ratio and high reliability $[9,10]$. Non-polarizable silver/silver chloride $(\mathrm{Ag} / \mathrm{AgCl})$ electrodes and conductive gels or pastes containing chloride ions are often used in these applications and referred to as 'wet' electrodes. The conductive gel forms an ionic path and construct a non-polarizable electric double layer on the electrode surface, which minimizes the polarization potential and ensures smooth baseline in EEG signal recording. Moreover, the gel-based electrode/scalp impedance has been proved to be very stable and tolerant of head movement, because the conductive gel can penetrate the hair, then conform to the scalp surface and wetting of the high impedance skin stratum corneum, even possibly penetrate into the inner layer of skin through sweat glands and pores. However, the setup of gel-based electrodes usually is time-consuming, including skin preparation (i.e. cleaning and abrasion of the skin) and conductive gels application [11]. Even worse, conductive gels dirty and mess the hair, and may cause discomfort for the users $[12,13]$. Therefore, these inconvenience and discomfort issues severely limit emerging EEG-based applications.

To overcome these problems, many efforts have been made to develop gel-free electrodes in recent years. The gel-free electrodes, usually referred to as 'dry' electrodes, consist of an electronic conductor with no conductive gel between electrode and scalp, such as inert metallic pins or tips [14-18], comb-like conductive polymer elastomer [19, 20] and flexible metalcoated bristles $[21,22]$. These dry electrodes actually still maintain a very tiny amount of electrolyte such as perspiration and moisture at the electrode/scalp interface. Nevertheless, as no conductive gel or paste application is needed, the 'dry' electrodes are bringing a significant 
improvement over the 'wet' electrodes for its quick setup, self-application, and cleanliness.

However, the absence of the conductive gel always leads to relative high impedance (i.e. several hundreds $\mathrm{k} \Omega$ or higher), due to the less effective ionic conductive path and interface double layer. A Multi-tips based dry electrode of the size of a US 5c coin was proposed by Matthews R. et al., with the contact impedance between the scalp and each tip being as high as 10 $\mathrm{M} \Omega$ [18]. A flexible, low-cost electrode made of polymer silver-coated bristles approximately the size of a toothbrush developed by Cristian Grozea et al. and an initial impedance of $80 \mathrm{k} \Omega$ was reported that deteriorated to $150 \sim 200 \mathrm{k} \Omega$ after 10 months of use [21]. Cognionics Inc. claims that the contact impedance of their flexible dry EEG electrodes is in the range of $100 \mathrm{k} \Omega$ to $2000 \mathrm{k} \Omega$ [23]. The motion artefact often arises from the disturbance at the electrode/skin interface [13]. Therefore, dry electrodes of high impedance are more sensitive to motion artefacts because of lack of enough electrolyte at the electrode/skin interface. In addition, the high impedance also tend to lead to unstable electrode potential. All of these cause poor signal quality [24-28].

Active dry electrodes with high input impedance preamplifiers inside were developed to alleviate the poor signal $[14,16,19,29-31]$. Since the active electrodes can convert the high electrode/skin impedance into a low impedance output, the signal quality is less dependent on the electrode/skin impedance [32]. Although active electrodes are less affected by environmental noise, they are still susceptible to movement artifacts. In addition, the active electrodes are usually bulky and expensive [25].

In order to overcome the problems of 'wet' and 'dry' electrodes, the 'quasi-dry' electrode 
concept was developed $[25,33]$. The working principle of 'quasi-dry' electrodes is to release electrolyte fluid by imposing a pressure on a saline reservoir instead of the present of conductive gels. Although the 'quasi-dry' electrodes demonstrate several merits over 'dry electrodes', but there are still some technical problems as follows. Firstly, it needs an additional pressure to enable continuously releasing the electrolyte fluid; Secondly, electrode deterioration, such as electrode deformation and electrode coating failure, can happen under long-term pressure. Moreover, it is very difficult to achieve uniform pressure. The non-uniform pressure brings uncontrolled, unexpected moistener release, thus leading to signal instability [25]. Finally, it is noteworthy that the EEG test results were not very sound, as the reported data were from only one participant. An alternative solution was proposed by Martins et al to avoid the pressure related issues, which was similar to "felt-pen" concept [34]. The concept employed a specifically developed polymer wick to achieve continuous and stable delivery of liquid without external pressure. Unfortunately, the electrochemical performance and EEG signal quality has not been reported.

In this study, we proposed a novel porous ceramic-based semi-dry electrode prototype aiming to overcome the problems of the 'quasi-dry' electrode. The semi-dry electrodes consist of sintered $\mathrm{Ag} / \mathrm{AgCl}$ electrode and saline in reservoir, which ensures a stable non-polarization electrode interface and an ionic conductive path. Similar to the "polymer wick" electrode concept, the semi-dry electrodes enable release a small amount of saline solution in a controlled and sustained manner, which achieves by the assistance of capillary forces in the porous ceramic pillars. It is clear that the semi-dry electrodes eliminate the inconvenience of using 
conductive gel. Further, the semi-dry electrodes retain the non-polarizable electrode/electrolyte interface, which can minimize polarization potential, then allows a smooth baseline in EEG recording. It also facilitates DC coupling of an amplifier.

To systematically evaluate the performance of the proposed semi-dry electrode, we conducted a series of electrochemical investigations including electrode/scalp impedance and electrode polarization performance. The performance was further assessed in a human EEG study: 10 subjects were recruited to participate in several classical EEG paradigms such as the eyes open/closed, as well as the SSVEPs.

\section{Materials and Methods}

\subsection{Semi-dry electrodes and headset}

The design of the semi-dry electrode prototype is illustrated in Figures 1A and 1B. The semi-dry electrode includes five porous ceramic pillars (i), a built-in reservoir (ii), $500 \mu \mathrm{L}$ $3.5 \%$ saline solution (iii) and sintered $\mathrm{Ag} / \mathrm{AgCl}$ electrode (iv). The aluminum oxide $\left(\mathrm{Al}_{2} \mathrm{O}_{3}\right)$ ceramic pillars were purchased from Suzhou Greentek (China), considering its character of excellent mechanical properties (i.e. wearability, resistance to compression) and good hydrophilic and permeability performances. The physical dimensions of the porous ceramic pillars are $\Phi 1.2 \mathrm{~mm} \times 7.0 \mathrm{~mm}$ with a few micrometer pores structure (Figure 1C). Using the capillary force of the porous ceramics, the semi-dry electrode enables continuous release of saline solution from the built-in reservoir at the rate of $10 \sim 20 \mu \mathrm{L} / \mathrm{h}$. Sintered $\mathrm{Ag} / \mathrm{AgCl}(\Phi 6.0$ $\mathrm{mm}, 1.0 \mathrm{~mm}$ thick, Greentek, China) were chosen as electrode material, considering its 
character of non-polarization, electrode potential stability, and low noise properties.

In the present study, the semi-dry electrodes were assembled into a customized 9-channel headset (shown in Figure 1D) for a series of in-vivo measurements including the electrode/scalp impedance tests and EEG signal recording. The semi-dry headset consists of ten semi-dry electrodes placed at $\mathrm{O} 1, \mathrm{Oz}, \mathrm{O} 2, \mathrm{P} 3, \mathrm{Pz}, \mathrm{P} 4, \mathrm{C} 3, \mathrm{Cz}, \mathrm{C} 4$ and $\mathrm{Fz}$ (as ground electrode). A comparison test was conducted between the semi-dry and the conventional 'wet' electrodes that consisted of sintered $\mathrm{Ag} / \mathrm{AgCl}$ electrodes and conductive gel (Greentek, China). A 'wet' electrode cap was worn and the semi-dry electrode headset was placed over the 'wet' electrode cap.

\subsection{Subject and ethical information}

Ten subjects (four females, age between 21 and 34 years) were enrolled in the study. All of them were free of medication, had normal vision or vision corrected to normal, and no history of central nervous system abnormalities. The study was conducted in accordance with the Declaration of Helsinki and approved by the local ethics committee of Wuhan University. All subjects participated in the semi-dry electrode/scalp impedance tests first, followed by the EEG signal evaluation tests. Two of them also participated in an $8 \mathrm{~h}$ electrode/scalp impedance test to evaluate the long-term stability.

\subsection{Electrode/scalp impedance tests}

The semi-dry electrodes were fixed on subjects' scalp by a customized 9-channel headset as described in section 2.1 , the electrode $\mathrm{Cz}$ was used as the reference electrode in all impedance tests. After electrode setup, the nine semi-dry electrode pairs (vs $\mathrm{Cz}$ ) impedance 
value at $10 \mathrm{~Hz}$ were recorded immediately separately. No any skin preparations, such as cleaning and removal the stratum corneum by some skin prep gels or alcohols, were made for any subject. All impedances presented in the study were measured by an impedance/gain-phase analyzer (Solartron 1260, UK) using $50 \mathrm{mV}$ (rms) AC sinusoid signal at a frequency range from $100 \mathrm{kHz}$ to $1 \mathrm{~Hz}$ by the two-electrodes methods.

The influence of the semi-dry electrode structure on semi-dry electrode/scalp impedance at $100 \mathrm{kHz}$ and $10 \mathrm{~Hz}$ was also investigated using the following setup (Figure 2A): i) the semidry electrode (semi-dry electrode structure $\mathrm{S}+$ sintered $\mathrm{Ag} / \mathrm{AgCl}$ electrode $\mathrm{E}$ ) on hairy scalp; ii ) the semi-dry electrode (semi-dry electrode structure $\mathrm{S}+$ sintered $\mathrm{Ag} / \mathrm{AgCl}$ electrode $\mathrm{E}$ ) in $3.5 \%$ saline, and iii) sintered $\mathrm{Ag} / \mathrm{AgCl}$ electrode $(\mathrm{E})$ in $3.5 \%$ saline. The impedance at $10 \mathrm{~Hz}$ largely reflects the dominant impact of scalp, because of the capacitive property of the skin, the impedance diminishes at $100 \mathrm{kHz}$.

\subsection{Electrode polarization voltage measurement}

Open circuit potential (OCP) of semi-dry electrodes against a sintered $\mathrm{Ag} / \mathrm{AgCl}$ reference electrode ( $\Phi 8.5 \mathrm{~mm}, 1.0 \mathrm{~mm}$ thick, Greentek, China) were recorded in 3.5\% saline solution with a Keithley 2000 Mulitimeter (Keithley Instrument, Inc., USA) for $10 \mathrm{~min}$ at a sampling rate of $1 \mathrm{~Hz}$. Eight $\mathrm{Ag} / \mathrm{AgCl}$ sintered electrodes used in the semi-dry electrodes $(\Phi 6.0 \mathrm{~mm}$, 1.0mm thick, Greentek, China) were also tested for a comparison.

The two important parameters, equilibrium electrode potential and potential drift, were used to evaluate the electrode polarization characteristics. Equilibrium electrode potential was defined as the average potential value within $10 \mathrm{~min}$. Electrode drift was defined as the 
maximum electrode potential value minus the minimum electrode potential value within 10 $\min$.

\subsection{Long-term stability of semi-dry electrode impedance}

The semi-dry electrode/scalp impedance was measured with $1 \mathrm{~h}$ interval up to $8 \mathrm{~h}$. The four semi-dry electrodes at $\mathrm{Oz}, \mathrm{Pz}, \mathrm{C} 4$ and $\mathrm{Fz}$ were tested, with the semi-dry electrode $\mathrm{Cz}$ used as the reference electrode for all tests by the two-electrodes methods.

\subsection{EEG test protocol and signal evaluation}

In order to validate the semi-dry electrode performance, EEG signals from both of the wet electrodes and the semi-dry electrodes were recorded simultaneously at very close locations of each subject in their hairy scalp.

\subsubsection{Electrodes setup}

The electrode setup (Figure 3) consists of nine pairs of semi-dry and 'wet' electrodes placed at $\mathrm{O} 1, \mathrm{Oz}, \mathrm{O} 2, \mathrm{P} 3, \mathrm{Pz}, \mathrm{P} 4, \mathrm{C} 3, \mathrm{Cz}$ and C4. Semi-dry and 'wet' electrodes were mounted on separate customized headsets (see section 2.1). The center-to-center distance between each pair was approximately $2 \mathrm{~cm}$. Such distance should be reasonable to make a fair comparison, ensuring that the almost negligible effect on the EEG signal by their locations, while avoiding conductive gel spread to the semi-dry electrode sites. In addition, one 'wet' electrode placed at Fz was used as the ground electrode and another wet electrode placed on the right mastoid was used as the reference electrode. Each 'wet' electrode was filled with GT5 freeprep conductive gel (Greentek, China) using a blunted needle and syringe rocking or rotating to lower the impedance quickly. The 'wet' electrode-skin impedances were kept below $5 \mathrm{k} \Omega$ in the EEG 
recordings. Care was taken to avoid conductive gel spread to the semi-dry electrode sites. Almost few conductive gels were found at the semi-dry electrode sites after carefully examination.

A NeuroScan amplifier (SynAmp II, NeuroScan, Compumedics, USA) was used for EEG recording at a sampling rate of $1000 \mathrm{~Hz}$. In order to compare the two types of electrodes, no hardware built-in spectral filters were applied, hereby all DC components as well as the powerline noises were honestly recorded.

\subsubsection{EEG recording protocol}

The experimental protocol lasted for about $30 \sim 45$ minutes. Subjects were seated in a comfortable chair before a 19-inch ViewSonic E90fB CRT monitor in a sound-attenuated, dimly lit, and electrically shielded room. The monitor's screen resolution was $1024 \times 768$ and the refresh rate was set as $60 \mathrm{~Hz}$. The following two experimental paradigms were employed:

\section{(1) Eyes open/closed}

Subjects were instructed to perform an eyes open/closed task for 10 minutes. Specifically, they were required to first close their eyes for 5 minutes, and then open their eyes to read a history book for 5 minutes. The light of the room was switched on during this part of the experiment to provide a proper luminance level for reading.

(2) SSVEPs

Subjects were presented with four white squares flickering at different frequencies $(20 \mathrm{~Hz}$, $15 \mathrm{~Hz}, 12 \mathrm{~Hz}$, and a non-flicker). The experiment consisted of 32 trials, with each flicker serving as the attentional target for 8 trials. The order of the trials was randomized and the 
subjects were cued to the to-be-attended flicker visually, by a 1-second pre-trial cue screen with all the four flickers except the target flicker in red. Each trial lasted for 7 seconds and the intertrial interval was 4 seconds.

\subsubsection{Data preprocessing and analyses}

The eyes open/closed data were compared mainly based on their spectra using Fourier transform. Specifically, the EEG data were segmented into 1-second epochs and the singleepoch spectra were averaged within each condition and then contrasted between the two conditions. A clear peak around the alpha range $(8-12 \mathrm{~Hz})$ was expected, over the parietaloccipital area. The SSVEPs data were analyzed based on their spectra as well. The spectra were obtained using Fourier transform on 1-second epochs, resulting in a spectral resolution of $1 \mathrm{~Hz}$. The EEG data from 1 second to 7 seconds after the flickering onset were used. The spectral responses at the three flickering frequencies (i.e. $12 / 15 / 20 \mathrm{~Hz}$ ) were the frequencies of interest.

To have an overview of the signal similarities between the two types of electrodes, the signals were subjected to the temporal correlation analysis using Pearson correlation. The temporal correlation analysis was computed separately for the EEG data collected during the eyes open/closed paradigm and the SSVEPs paradigm. The analysis was carried out using a time window of 5 seconds ( $20 \%$ overlap between continuous time windows) and the reported results were the averages over all possible time windows.

To quantitatively assess the paradigm-specific EEG signal qualities, the signal-to-noise ratios (SNRs) were computed based on the spectral responses (SRs), as following: 


$$
S N R=20 * \log _{10}\left[\frac{\text { mean }\left(S R_{\text {frequencies of interest }}\right)}{\text { mean }\left(S R_{\text {background }}\right)}\right]
$$

For the eyes open/closed paradigm, the SNRs were computed for the eyes closed condition and the frequency range of interest is $8 \sim 12 \mathrm{~Hz}$ (i.e. the alpha band). For the SSVEPs paradigm, the SNRs were computed separately for the three conditions, where the attended flickering frequencies of these conditions were considered as the frequencies of interest (i.e. 12 / 15 / 20 $\mathrm{Hz}$ ). The background EEG activities were defined as the EEG spectral responses of $3 \sim 40 \mathrm{~Hz}$, while the frequencies of interest are excluded.

\section{Results and discussion}

\subsection{Semi-dry electrode/scalp impedance}

Electrode impedance at the electrode-skin interface is a key parameter to obtain a reliable EEG signal transfer, because the lower the interfacial impedance the more immune the signal will be to noise, electromagnetic interference and movement artifacts. The mean and standard deviations of the semi-dry electrode/scalp impedance of all nine different positions for 10 subjects are shown in Figure 4A, and the values were $42.1 \pm 16.4 \mathrm{k} \Omega(\mathrm{O} 1) \sim 51.4 \pm 21.8 \mathrm{k} \Omega$ (O2). It should be pointed out that the impedance measured in this study resulted from a pair of semi-dry electrodes at $10 \mathrm{~Hz}$. If we report it as a single electrode impedance tested by a EEG amplify, the impedance can be half $(21.1 \pm 8.2 \mathrm{k} \Omega \sim 25.7 \pm 10.9 \mathrm{k} \Omega)$ approximately. Surprisingly, the study found that the impedance variation for each subject between all nine electrodes positions except the electrodes placed at $\mathrm{O} 2$ were within $5 \mathrm{k} \Omega$, which indicates that 
the impedance at different positions for each person was very close. Therefore, it would be very beneficial to the common mode rejection of the EEG amplifier.

The mean and standard deviations of the semi-dry electrode/scalp impedance for the ten subjects is presented in Figure 4B. The average impedance of the ten subjects was $44.4 \pm 16.9$ $\mathrm{k} \Omega$, ranging from $21.87 \pm 2.9 \mathrm{k} \Omega$ (subject 8 , male, with short, thin and rare hair) to $69.59 \pm$ $5.02 \mathrm{k} \Omega$ (subject 10, female, with long, thick and dense hair). Likewise, the average of a single (not a pair) semi-dry electrode for ten subjects can be estimated as $22.2 \pm 8.5 \mathrm{k} \Omega$ approximately. The obvious individual variations of the impedance can be mainly explained by different skin conditions of the participant including size of sweat glands and pores, the thickness of skin stratum corneum, and the length, thickness, and density of hair. Subjects with long, thick and dense hair were found to have higher impedance values and vice versa. This phenomenon agrees well with previous report [35]. This individual variation is also associated with noneven pressure force applied by semi-dry electrodes to individuals because the headset design does not well fit to each subject head shape [36]. Next, we will further improve the design of the headset to better fit the individuals' scalp.

Electrode-scalp impedance between our semi-dry electrodes and literature reported wet electrodes or dry electrodes are listed in Table 1. The dry electrode/scalp interfacial impedance is in a range of the hundreds of $\mathrm{k} \Omega$ compared to $5 \sim 10 \mathrm{k} \Omega$ for gel-based electrodes [23]. To the best of our knowledge, the lowest 'dry' electrode/scalp impedance for passive dry electrode is $80 \mathrm{k} \Omega$ reported by Cristian Grozea et al [21]. Although the impedance of the semi dry electrode $(\sim 11-35 \mathrm{k} \Omega$, Fig 4B) is higher than that of the gel-based electrodes $(5 \sim 10 \mathrm{k} \Omega)$, the 
impedance is significantly reduced compared with the existing dry electrodes $(80 \sim 2000 \mathrm{k} \Omega)$.

Last but not least, the semi-dry electrode/scalp impedance tests in this study were immediately performed on unprepared scalps after setup $(n=10)$, unlike other impedance results which were reported based on one or two subjects on the bare skin (such as forearm and forehead) or prepared hairy scalp [33, 37-40]. Therefore, the results here are expected to represent the impedance of the semi-dry electrodes in practical situations.

\subsection{Effect of semi-dry electrode structure on electrode/scalp impedance}

Comparing the impedance of the semi-dry electrodes to the sintered $\mathrm{Ag} / \mathrm{AgCl}$ electrode while they both were in contact to $3.5 \%$ saline (see setting ii and setting iii, Figure $2 \mathrm{~A}$ ), the impedance both at $100 \mathrm{kHz}$ and $10 \mathrm{~Hz}$ have increased by approximately $2000 \Omega$ (Figure $2 \mathrm{~B}$ ). It clearly reveals that the porous ceramic pillars structure contributes about $2000 \Omega$ pure resistance that is irrelevant to measuring frequencies, and the impedance contributed by the ceramic structure is dominant while the electrodes to the saline interface impedance can be neglected.

Looking at the impedance results of setting $\mathrm{i}$ and setting ii (Figure 2A), which is a comparison of semi-dry electrodes in contact to the saline and to scalp, a significant elevated impedance appeared at $10 \mathrm{~Hz}$, of $37.7 \mathrm{k} \Omega$ (from $2.1 \pm 0.2 \mathrm{k} \Omega$ to $39.8 \pm 3.4 \mathrm{k} \Omega$ ) on scalp (Figure 2B). At $100 \mathrm{kHz}$, the semi-dry electrodes/scalp impedance was $570 \Omega$ higher than that of semidry electrodes/saline impedance (Figure 2B). The increase in impedance was mainly contributed by the ceramic pillars and scalp interface, influenced by contact condition between them, and also affected by high impedance of the skin stratum corneum and availability of fluid 
electrolyte. An increase in skin impedance with a decrease of frequency is due to capacitive characteristics of the skin, which well explains the significant increase in semi-dry electrode/scalp impedance at $10 \mathrm{~Hz}$. It should be pointed out that the contribution of the resistance of ionic conductivity in porous ceramic pillars can be ignored compared with that impedance generated on the boundary of the semi-dry electrode tips and scalp at $10 \mathrm{~Hz}$, which is meaningful to EEG recordings.

\subsection{Effect of semi-dry electrode structure on electrode polarization voltage}

The stability of electrode polarization voltage directly affects the baseline stability and low frequency noise in EEG recording, especially for DC EEG recording. Acquiring high quality EEG signals depends on good non-polarizable electrodes, which are characterized by minimum difference of equilibrium potential between electrodes and small potential drift over time. Although it is very important, the electrode polarization performance is often neglected in the development of new gel-free EEG electrodes.

The open circuit potential (OCP) of semi-dry electrodes and sintered $\mathrm{Ag} / \mathrm{AgCl}$ electrodes after 10 min stabilization in $3.5 \%$ saline solution are displayed in Figure 5. The box-andwhisker plot comparing the equilibrium potential between semi-dry electrodes and $\mathrm{Ag} / \mathrm{AgCl}$ electrodes are shown in Figure 6. The semi-dry electrode can be seen as a sintered $\mathrm{Ag} / \mathrm{AgCl}$ electrode plus a container with 5 pillars of porous ceramic (Figures 1A and 1B). The sintered $\mathrm{Ag} / \mathrm{AgCl}$ electrodes resulted in a low and stable potential, with a mean equilibrium electrode potential of $0.323 \mathrm{mV}$ and a standard deviation (STD) of $0.186 \mathrm{mV}$. The OCP of the semi-dry electrode was higher, with a mean equilibrium electrode potential of $1.404 \mathrm{mV}$ with STD 0.203 
$\mathrm{mV}$. The maximum difference of equilibrium potential between eight semi-dry electrodes was $0.579 \mathrm{mV}$, and meanwhile that of sintered $\mathrm{Ag} / \mathrm{AgCl}$ electrodes was $0.453 \mathrm{mV}$. The potential drift of the semi-dry electrodes was $29 \pm 14 \mu \mathrm{V}$ within $10 \mathrm{~min}$, while the sintered $\mathrm{Ag} / \mathrm{AgCl}$ electrodes was $46 \pm 32 \mu \mathrm{V}$. The variation of equilibrium potential and potential drift is as good as that of the sintered $\mathrm{Ag} / \mathrm{AgCl}$ electrodes. Therefore, the semi-dry electrode structure has little effect on the polarization voltage.

\subsection{Long-term stability of semi-dry electrodes in vivo}

The long-term stability of the semi-dry electrode performance determines a time window for an application. The semi-dry electrode/scalp impedance, an important parameter for electrode-scalp interface, was recorded to evaluate the long-term performance. The impedance at $10 \mathrm{~Hz}$ up to $8 \mathrm{~h}$ is shown in Figure 7. A pair of the semi-dry electrode/scalp impedance increased by approximately $20 \mathrm{k} \Omega$ for the two subjects in $8 \mathrm{~h}$. In previous reports, the impedance of wet electrodes increased from $5 \mathrm{k} \Omega$ to $15 \mathrm{k} \Omega$ within $5 \mathrm{~h}$ after gel application due to gradual drying over time [39]. The impedance variation with time of semi-dry electrodes was similar to that of wet electrodes, which may be attributed to the release rate of saline slowing down gradually and the continuous evaporation of the saline. These results suggest that the semi-dry electrodes can effectively record the EEG signals up to $8 \mathrm{~h}$.

\subsection{EEG signal evaluation}

An example of simultaneous wet and semi-dry recordings in the eyes open/closed paradigm at all test positions in time domain are presented in Figure 8. The EEG records of both the semi-dry and the wet electrodes clearly show strong alpha oscillations under the eyes 
closed condition. Furthermore, the EEG results between the two types of electrodes were fairly similar while they are located in adjacent positions. The spectra of eyes open /closed at the electrode position $\mathrm{Pz}$ (both wet and semi-dry) from a representative subject are illustrated in Figures 9A and 9B, showing similar spectral peaks at 8-12 Hz.

The spectra of the SSVEPs responses at the electrode position $\mathrm{Oz}$ from a representative subject is illustrated in Figures 9C and 9D. For both types of the electrodes, clear spectral peaks were found at the three flickering frequencies, and the different attention conditions indeed associated with the peak of SSVEPs responses at the corresponding frequencies (except for the attending to the non-flicker condition, which did not show strong peaks at these frequencies).

To have a comparison of the signal similarities between the wet and semi-dry electrodes, the signals were subjected to the temporal correlation analysis using Pearson correlation. For the eye open/closed paradigm, the average temporal cross-correlation between semi-dry/wet electrodes was $0.938 \pm 0.037$ across the nine recording electrode pairs with ten subjects, with the highest at $\mathrm{Oz}(0.997)$ and the lowest at C3 (0.883) (Figure 10A). For the SSVEPs paradigm, the average temporal cross-correlation between semi-dry/wet electrodes was $0.937 \pm 0.027$ $(n=10)$ across the nine recording electrode pairs with ten subjects, with the highest at $\mathrm{Oz}(0.993)$ and the lowest at C3 (0.904) (Figure 10B). The correlation results suggest that signals of the semi-dry and wet electrodes pairs were highly similar, and the correlation coefficients of all positions matches or exceeds the reported levels of most existing dry electrodes, ranging from 0.32 to $0.94[17,25,36,41-45]$. Furthermore, the correlation coefficients of all positions were around 0.9 , suggesting that the semi-dry electrodes' performances were robust against factors 
such as hair thickness.

In order to assess the paradigm-specific EEG signal qualities quantitatively, the signal-tonoise ratios (SNRs) were computed based on the spectral responses (SRs). The SNRs over all subjects for occipital domain, namely $\mathrm{O} 1, \mathrm{Oz}$ and $\mathrm{O} 2$, are presented in Figure 11. When comparing the signal recorded by the adjacent semi-dry/wet electrode pairs, the SNRs for the semi-dry electrodes were quantitatively comparable with the wet $\mathrm{Ag} / \mathrm{AgCl}$ electrodes. Moreover, all the paradigm-specific SNRs difference between two types of electrodes was not significant (except $12 \mathrm{~Hz}$ SSVEP at O2, $t(9)=-2.749, p=0.023$ ). This exception may be related to the $12 \mathrm{~Hz}$ stimulation frequency.

Overall, the in vivo EEG recording demonstrated that the semi-dry electrodes were capable of acquiring reliable EEG signals as compared to those of the commercial $\mathrm{Ag} / \mathrm{AgCl}$ wet electrodes, validating the new electrode design for EEG monitoring.

\subsection{Advantages and further improvements of semi-dry electrode}

We have developed a novel passive, porous ceramic-based semi-dry electrode prototype, and compared it with the conventional gel-based $\mathrm{Ag} / \mathrm{AgCl}$ electrode using a 9-channel headset. The novel passive semi-dry electrodes have many outstanding advantages as follows.

- Minimize electrode polarization

The semi-dry electrodes have shown non-polarization characteristics the same as traditional $\mathrm{Ag} / \mathrm{AgCl}$ electrodes. The semi-dry electrodes maintain the non-polarizable electrode/electrolyte interface, which can minimize polarization potential. This allows a smooth baseline in EEG recording and facilitates EEG recording in low frequency range. 
- Low and stable electrode/scalp impedance

Compared with dry electrodes, the semi-dry electrodes have a low and stable semi-dry electrode/scalp impedance. This can be well explained by the follow reasons. Firstly, it provides an ionic current path by slowly releasing a small amount of saline into the scalp surface, and wetting the scalp surface; Secondly, the small amount of saline can even penetrate through the skin surface of sweat glands or pores into the inner layer of the skin, thus bypassing partly the high impedance skin stratum corneum to form an ion conductive path to further reduce the electrode scalp interface impedance.

\section{- Reliable EEG signal}

The semi-dry electrodes build a reliable electrical path between electrode-scalp, can minimize electrode polarization and lead to relative low and stable electrode-scalp impedance. As a result, the semi-dry will have good quality signal that is comparable to the 'wet' electrode. The motion artefact often arises from the disturbance at the electrode/skin interface [13]. The effect of semi-dry electrodes' saline buildup is to act as a physical "shock absorber", which is similar to, but less effective than, the action of conductive gel used in wet electrodes. This suggest that the semi-dry electrodes maybe less sensitive to motion artefacts compared with dry electrode.

\section{- Passive design}

Signal noise caused by high contact impedance can be alleviated by using preamplifier integrated to electrodes (active dry electrodes), or shielding of the electrode wires. However, the active electrodes are usually bulkier and more expensive. Due to the greater improvement 
in impedance, the semi-dry electrodes would not require a preamplifier. The passive design favors wearability and simple manufacture.

- Not heavily dependent on the contact pressure

A relative high pressure is required to ensure a proper impedance for dry electrodes, which is a big problem as it could cause pain. The effect of pressure largely on dry electrode impedance has already been reported [46-48], and results showed that impedance decreases with an increase in contact pressure. However, the long-term pressure applied through dry electrodes usually causes pain and discomfort $[47,48]$. The semi-dry electrodes with relative low impedance is not heavily dependent on the pressure of the support system anymore, which will improve the comfortable level of users.

- User friendly

As semi-dry electrodes eliminate the inconvenience of using conductive gel, the 'dry' electrodes are bringing a significant improvement over the 'wet' electrodes for its quick and simple setup, self-application, and cleanliness for users. No doubt, the improvement of user friendly is very crucial to promoting the rising real-world EEG applications and wearable EEGbased devices.

- Further improvements

In spite of the good performance of the new semi-dry electrodes, some technical problems related to the electrode and electrode headset are worth further investigation. For example, it is necessary to investigate the influence of various ceramic materials, porosity and electrolyte concentration on the electrode performance. Furthermore, the semi-dry electrodes still need to 
improve the convenience for users because of the need to add saline solution. New electrolyte materials and electrolyte permeable material would facilitate automatic "charge - discharge" electrolyte, which is an exciting subject to be investigated further.

\section{Conclusions}

Novel semi-dry electrodes embedded in a headset for EEG recording, aiming to overcome the hurdles of current wet and dry electrodes, has been investigated. The semi-dry electrodes were fabricated and evaluated on ten subjects' scalps. The semi-dry electrodes are able to release a small amount of saline solution with assistance of capillary force through porous ceramic pillars, thus eliminating the use of skin preparation and gel application. The semi-dry electrodes have demonstrated many advantages including quick setup, self-application, up to 8 $\mathrm{h}$ usage window and cleanliness for users.

The electrochemical tests showed that the semi-dry electrodes/scalp impedance was relatively low and stable compared with existing 'dry' electrodes. The semi-dry electrodes polarization voltage was stable and equivalent to that of the 'wet' electrodes. The EEG signal tests have showed that the semi-dry electrode was able to acquire reliable EEG signals similar to those of the commercial gel-based $\mathrm{Ag} / \mathrm{AgCl}$ electrodes. The semi-dry electrodes have a great potential to be applied to real-world practical EEG applications, such as brain-computer interfaces and wearable devices.

\section{Acknowledgements}


The authors gratefully acknowledge the support of the National Key Technology R\&D Program (2012BAI16B02), the National Science Foundation of China under grant \#61101151 and Tsinghua University Initiative Scientific Research Program (2014z21043). The authors are also very grateful to Fei Wang (Department of Psychology, School of Social Sciences, Tsinghua University) and Jingjing Chen (Department of Biomedical Engineering, School of Medicine, Tsinghua University) to provide technical support on EEG signal recording. 


\section{References}

[1] P. Reis, F. Hebenstreit, F. Gabsteiger, V. von Tscharner, M. Lochmann, Methodological aspects of EEG and Body dynamics measurements during motion, Frontiers in Human Neuroscience, 8(2014).

[2] F. Tey, S.T. Lin, Y.Y. Tan, X.P. Li, A. Phillipou, L. Abel, Novel Tools for Driving Fatigue

Prediction: (1) Dry Eeg Sensor and (2) Eye Tracker, in: D.D. Schmorrow, C.M. Fidopiastis (Eds.), Foundations of Augmented Cognition: 7th International Conference, AC 2013, Held as Part of HCI International 2013, Las Vegas, NV, USA, July 21-26, 2013 Proceedings, Springer Berlin Heidelberg, Berlin, Heidelberg, 2013, pp. 618-27.

[3] M. Abo-Zahhad, S.M. Ahmed, S.N. Abbas, State-of-the-art methods and future perspectives for personal recognition based on electroencephalogram signals, Biometrics, IET, 4(2015) 179-90.

[4] C. Jin-Chern, K. Li-Wei, L. Chin-Teng, H. Chao-Ting, J. Tzyy-Ping, L. Sheng-Fu, et al., Using novel MEMS EEG sensors in detecting drowsiness application, Biomedical Circuits and Systems Conference, 2006 BioCAS 2006 IEEE2006, pp. 33-6.

[5] J.-A. Micoulaud Franchi, P.A. Geoffroy, G. Fond, R. Lopez, S. Bioulac, P. Philip, EEG Neurofeedback treatments in children with ADHD: An updated meta-analysis of Randomized Controlled Trials, Frontiers in Human Neuroscience, 8(2014).

[6] B. Zoefel, R.J. Huster, C.S. Herrmann, Neurofeedback training of the upper alpha frequency band in EEG improves cognitive performance, NeuroImage, 54(2011) 1427-31.

[7] L. Astolfi, F. De Vico Fallani, F. Cincotti, D. Mattia, L. Bianchi, M.G. Marciani, et al., 
Neural Basis for Brain Responses to TV Commercials: A High-Resolution EEG Study, Neural Systems and Rehabilitation Engineering, IEEE Transactions on, 16(2008) 522-31.

[8] G. Vecchiato, L. Astolfi, F. De Vico Fallani, J. Toppi, F. Aloise, F. Bez, et al., On the Use of EEG or MEG Brain Imaging Tools in Neuromarketing Research, Computational Intelligence and Neuroscience, 2011(2011) 12.

[9] V. McAdams, Bioelectrodes, in: J.G. Webster (Ed.) Encyclopedia of Medical Devices and Instrumentation, Wiley, New York, 1988, pp. 120-66.

[10] P. Tallgren, S. Vanhatalo, K. Kaila, J. Voipio, Evaluation of commercially available electrodes and gels for recording of slow EEG potentials, Clinical Neurophysiology, 116(2005) 799-806.

[11] M. Teplan, Fundamentals of EEG measurement, Measurement Science Review, 2(2002) $1-11$.

[12] L. Lun-De, L. Chin-Teng, K. McDowell, A.E. Wickenden, K. Gramann, J. Tzyy-Ping, et al., Biosensor Technologies for Augmented Brain\&\#x2013;Computer Interfaces in the Next Decades, Proceedings of the IEEE, 100(2012) 1553-66.

[13] A. Searle, L. Kirkup, A direct comparison of wet, dry and insulating bioelectric recording electrodes, Physiological Measurement, 21(2000) 271.

[14] C. Guger, G. Krausz, B.Z. Allison, G. Edlinger, Comparison of dry and gel based electrodes for P300 brain-computer interfaces, Frontiers in Neuroscience, 6(2012).

[15] L.-D. Liao, I.-J. Wang, S.-F. Chen, J.-Y. Chang, C.-T. Lin, Design, Fabrication and Experimental Validation of a Novel Dry-Contact Sensor for Measuring 
Electroencephalography Signals without Skin Preparation, Sensors, 11(2011) 5819.

[16] E.W. Sellers, P. Turner, W.A. Sarnacki, T. McManus, T.M. Vaughan, R. Matthews, A Novel Dry Electrode for Brain-Computer Interface, in: J.A. Jacko (Ed.) Human-Computer Interaction Novel Interaction Methods and Techniques: 13th International Conference, HCI International 2009, San Diego, CA, USA, July 19-24, 2009, Proceedings, Part II, Springer Berlin Heidelberg, Berlin, Heidelberg, 2009, pp. 623-31.

[17] P. Fiedler, P. Pedrosa, S. Griebel, C. Fonseca, F. Vaz, E. Supriyanto, et al., Novel Multipin Electrode Cap System for Dry Electroencephalography, Brain Topography, 28(2015) 647-56. [18] R. Matthews, P.J. Turner, N.J. McDonald, K. Ermolaev, T.M. Manus, R.A. Shelby, et al., Real time workload classification from an ambulatory wireless EEG system using hybrid EEG electrodes, Engineering in Medicine and Biology Society, 2008 EMBS 2008 30th Annual International Conference of the IEEE2008, pp. 5871-5.

[19] T.R. Mullen, C.A.E. Kothe, Y.M. Chi, A. Ojeda, T. Kerth, S. Makeig, et al., Real-time neuroimaging and cognitive monitoring using wearable dry EEG, Biomedical Engineering, IEEE Transactions on, 62(2015) 2553-67.

[20] Y.-H. Chen, M. de Beeck, L. Vanderheyden, E. Carrette, V. Mihajlović, K. Vanstreels, et al., Soft, Comfortable Polymer Dry Electrodes for High Quality ECG and EEG Recording, Sensors, 14(2014) 23758.

[21] G. Cristian, D.V. Catalin, F. Siamac, Bristle-sensors-low-cost flexible passive dry EEG electrodes for neurofeedback and BCI applications, Journal of Neural Engineering, 8(2011) 025008. 
[22] V. Kitoko, T.N. Nguyen, J.S. Nguyen, Y. Tran, H.T. Nguyen, Performance of dry electrode with bristle in recording EEG rhythms across brain state changes, Engineering in Medicine and Biology Society, EMBC, 2011 Annual International Conference of the IEEE2011, pp. 5962.

[23] M. Lopez-Gordo, D. Sanchez-Morillo, F. Valle, Dry EEG Electrodes, Sensors, 14(2014) 12847.

[24] M. Fatoorechi, J. Parkinson, R.J. Prance, H. Prance, A.K. Seth, D.J. Schwartzman, A comparative study of electrical potential sensors and $\mathrm{Ag} / \mathrm{AgCl}$ electrodes for characterising spontaneous and event related electroencephalagram signals, Journal of Neuroscience Methods, 251(2015) 7-16.

[25] A.R. Mota, L. Duarte, D. Rodrigues, A.C. Martins, A.V. Machado, F. Vaz, et al., Development of a quasi-dry electrode for EEG recording, Sensors and Actuators A: Physical, 199(2013) 310-7.

[26] Y.M. Chi, J. Tzyy-Ping, G. Cauwenberghs, Dry-Contact and Noncontact Biopotential Electrodes: Methodological Review, Biomedical Engineering, IEEE Reviews in, 3(2010) 10619.

[27] E.S. Kappenman, S.J. Luck, The Effects of Electrode Impedance on Data Quality and Statistical Significance in ERP Recordings, Psychophysiology, 47(2010) 888-904.

[28] A.-M. Tautan, V. Mihajlovic, Y.-H. Chen, B. Grundlehner, J. Penders, W. Serdijn, Signal Quality in Dry Electrode EEG and the Relation to Skin-electrode Contact Impedance Magnitude. 
[29] D.M.D. Ribeiro, L.S. Fu, L.A.D. Carlos, J.P.S. Cunha, A Novel Dry Active Biosignal Electrode Based on an Hybrid Organic-Inorganic Interface Material, Sensors Journal, IEEE, 11(2011) 2241-5.

[30] C. Fonseca, J.P.S. Cunha, R.E. Martins, V.M. Ferreira, J.P.M. de Sa, M.A. Barbosa, et al., A Novel Dry Active Electrode for EEG Recording, Biomedical Engineering, IEEE Transactions on, 54(2007) 162-5.

[31] P.-Y. Tsai, W. Hu, T.B. Kuo, L.-Y. Shyu, A portable device for real time drowsiness detection using novel active dry electrode system, Engineering in Medicine and Biology Society, 2009 EMBC 2009 Annual International Conference of the IEEE, IEEE2009, pp. 37758.

[32] S. Ahokas, J. Malmivuo, P. Kauppinen, Development of Low Noise Active Electrode for High-Resolution EEG, in: O. Dössel, W.C. Schlegel (Eds.), World Congress on Medical Physics and Biomedical Engineering, September 7 - 12, 2009, Munich, Germany: Vol 25/7 Diagnostic and Therapeutic Instrumentation, Clinical Engineering, Springer Berlin Heidelberg, Berlin, Heidelberg, 2009, pp. 876-9.

[33] H.-L. Peng, L. Jing-Quan, H.-C. Tian, Y.-Z. Dong, B. Yang, X. Chen, et al., A novel passive electrode based on porous Ti for EEG recording, Sensors and Actuators B: Chemical, 226(2016) 349-56.

[34] A.C. Martins, A. Moreira, A.V. Machado, F. Vaz, C. Fonseca, J.M. Nóbrega, Development of polymer wicks for the fabrication of bio-medical sensors, Materials Science and Engineering: C, 49(2015) 356-63. 
[35] K. Kleffner-Canucci, P. Luu, J. Naleway, D.M. Tucker, A novel hydrogel electrolyte extender for rapid application of EEG sensors and extended recordings, Journal of neuroscience methods, 206(2012) 83-7.

[36] A.M. Tautan, W. Serdijn, V. Mihajlovic, B. Grundlehner, J. Penders, Framework for evaluating EEG signal quality of dry electrode recordings, Biomedical Circuits and Systems Conference (BioCAS), 2013 IEEE2013, pp. 186-9.

[37] N.A. Alba, R.J. Sclabassi, M. Sun, X.T. Cui, Novel hydrogel-based preparation-free EEG electrode, Neural Systems and Rehabilitation Engineering, IEEE Transactions on, 18(2010) 415-23.

[38] L. Seungchan, S. Younghak, W. Soogil, K. Kiseon, L. Heung-No, Dry electrode design and performance evaluation for EEG based BCI systems, Brain-Computer Interface (BCI), 2013 International Winter Workshop on2013, pp. 52-3.

[39] L. Chin-Teng, L. Lun-De, L. Yu-Hang, I.J. Wang, L. Bor-Shyh, C. Jyh-Yeong, Novel Dry Polymer Foam Electrodes for Long-Term EEG Measurement, Biomedical Engineering, IEEE Transactions on, 58(2011) 1200-7.

[40] W. Zhou, R. Song, X. Pan, Y. Peng, X. Qi, J. Peng, et al., Fabrication and impedance measurement of novel metal dry bioelectrode, Sensors and Actuators A: Physical, 201(2013) 127-33.

[41] J.R. Estepp, J.C. Christensen, J.W. Monnin, I.M. Davis, G.F. Wilson, Validation of a Dry Electrode System for EEG, Proceedings of the Human Factors and Ergonomics Society Annual Meeting, 53(2009) 1171-5. 
[42] J.R. Estepp, J.W. Monnin, J.C. Christensen, G.F. Wilson, Evaluation of a Dry Electrode System for Electroencephalography: Applications for Psychophysiological Cognitive Workload Assessment, Proceedings of the Human Factors and Ergonomics Society Annual Meeting, 54(2010) 210-4.

[43] P. Fiedler, S. Griebel, P. Pedrosa, C. Fonseca, F. Vaz, L. Zentner, et al., Multichannel EEG with novel Ti/TiN dry electrodes, Sensors and Actuators A: Physical, 221(2015) 139-47.

[44] G. Gargiulo, P. Bifulco, A. McEwan, J. Tehrani, R. Calvo, M. Romano, et al., Dry electrode bio-potential recordings, Engineering in Medicine and Biology Society (EMBC), 2010 Annual International Conference of the IEEE2010, pp. 6493-6.

[45] G. Gargiulo, R.A. Calvo, P. Bifulco, M. Cesarelli, C. Jin, A. Mohamed, et al., A new EEG recording system for passive dry electrodes, Clinical Neurophysiology, 121(2010) 686-93.

[46] Y. Yamamoto, T. Yamamoto, Characteristics of skin admittance for dry electrodes and the measurement of skin moisturisation, Medical and Biological Engineering and Computing, 24 $71-7$.

[47] C. Grozea, G. Nolte, F. Popescu, Performance of novel dry electrode EEG cap for evoked potential and band-power activity detection, in: O. Dössel, W.C. Schlegel (Eds.), World Congress on Medical Physics and Biomedical Engineering, September 7 - 12, 2009, Munich, Germany: Vol 25/9 Neuroengineering, Neural Systems, Rehabilitation and Prosthetics, Springer Berlin Heidelberg, Berlin, Heidelberg, 2009, pp. 510-3.

[48] F. Popescu, S. Fazli, Y. Badower, B. Blankertz, K.-R. Müller, Single Trial Classification of Motor Imagination Using 6 Dry EEG Electrodes, PLoS ONE, 2(2007) e637. 
Table1 Electrode-scalp impedance between our semi-dry electrodes and literature reported wet electrodes or dry electrodes.

\begin{tabular}{|c|c|c|}
\hline \multicolumn{2}{|c|}{ Electrodes } & \multirow[t]{2}{*}{ Electrode/scalp impedance } \\
\hline Semi-dry & A pair of electrodes & \\
\hline electrode & A single electrode & $21.1 \pm 8.2 \mathrm{k} \Omega \sim 25.7 \pm 10.9 \mathrm{k} \Omega$ \\
\hline Dry electrode & A single electrode & $\begin{array}{l}\text { Dry electrode: several hundreds } \mathrm{k} \Omega \text { or higher } \\
\text { the lowest impedance: } 80 \mathrm{~K} \Omega(150-200 \mathrm{~K} \Omega \text { after } 10 \text { months of use) } \\
\text { [21] } \\
\text { Commercial dry electrode: } 100-2000 \mathrm{k} \Omega \text { (Cognionics Inc., US) } \\
\text { [23] }\end{array}$ \\
\hline Wet electrode & A single electrode & typically $5 \sim 10 \mathrm{~K} \Omega$ [23], below $5 \mathrm{k} \Omega$ by abrasive paste or gel \\
\hline
\end{tabular}



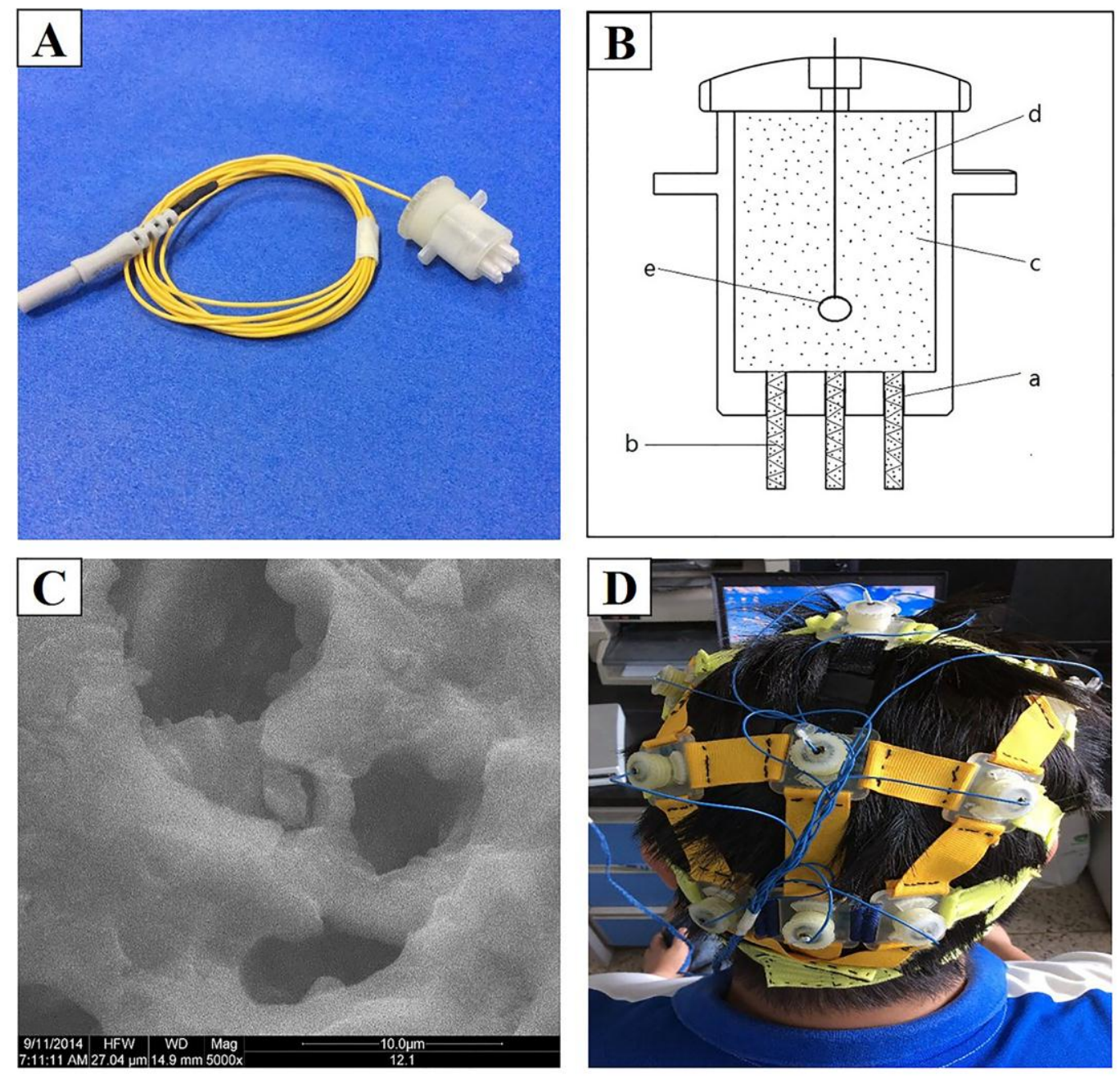

Figure 1 (A) Photo of a single semi-dry electrode. (B)Schematic diagram of the semi-dry electrode prototype, including porous ceramic pillars (i), a built-in reservoir (ii), 3.5\% saline solution (iii), and sintered $\mathrm{Ag} / \mathrm{AgCl}$ electrode (iv). (C) The SEM photo of porous ceramic with magnification 5000×, showed that porous structure with $3 \sim 6 \mu$ m diameter. (D) Photo of the headsets for semi-dry electrodes. 

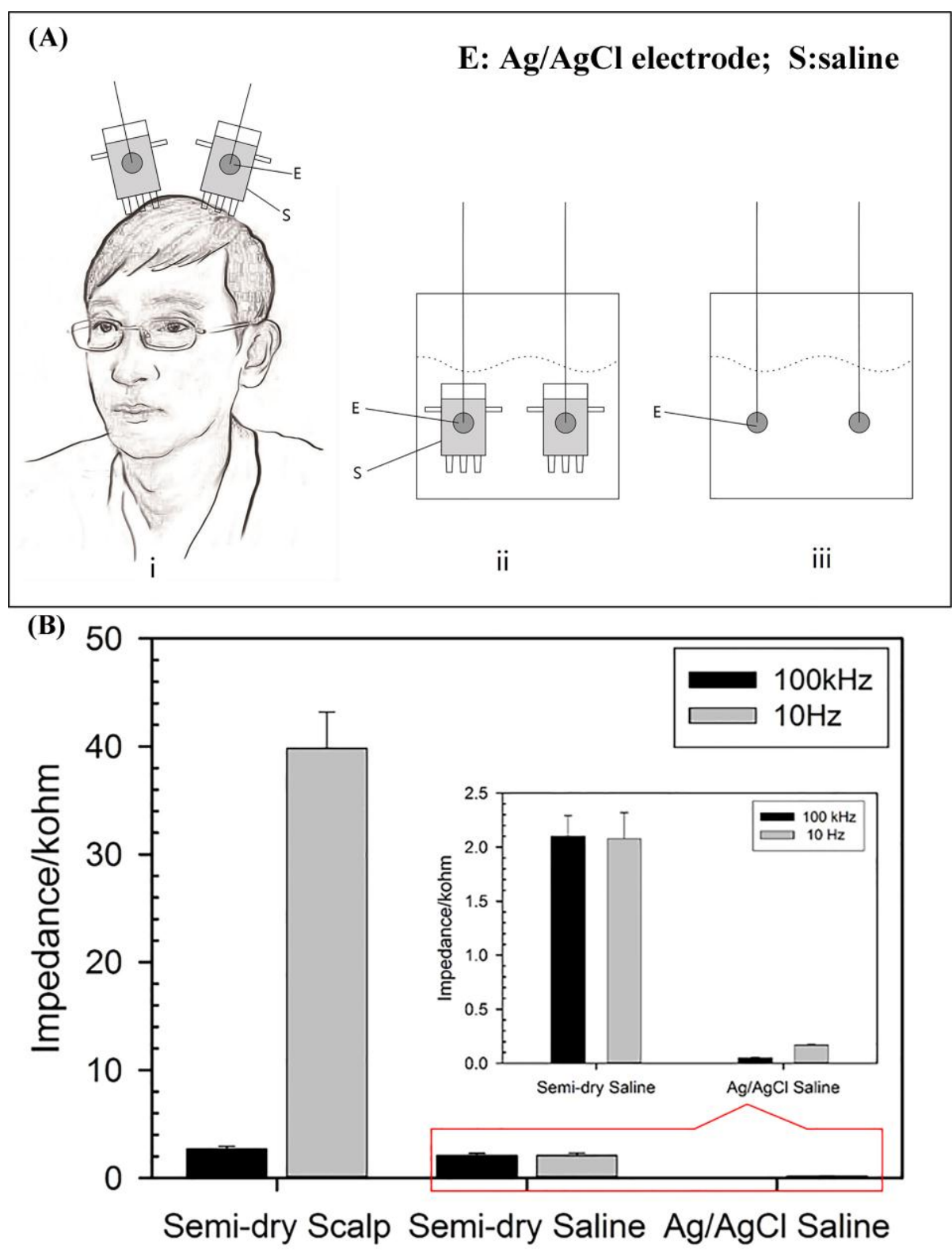

Figure 2 A comparison of three types of electrodes interface impedance at $100 \mathrm{kHz}$ and $10 \mathrm{~Hz}$. (A) The interface impedance test conditions: (i) semi-dry electrodes on subject's scalp, (ii) semi-dry electrodes in $3.5 \%$ saline solution and (iii) $\mathrm{Ag} / \mathrm{AgCl}$ electrodes (with same surface area as the semidry electrodes) in $3.5 \%$ saline solution, the electrode number of each type is four $(n=4)$; (B) The results of three types of electrodes interface impedance at $100 \mathrm{kHz}$ and $10 \mathrm{~Hz}$, and the inner inset is the magnification of semi-dry electrodes and $\mathrm{Ag} / \mathrm{AgCl}$ (used in the semi-dry electrodes) interface impedance tested in $3.5 \%$ saline solution. 


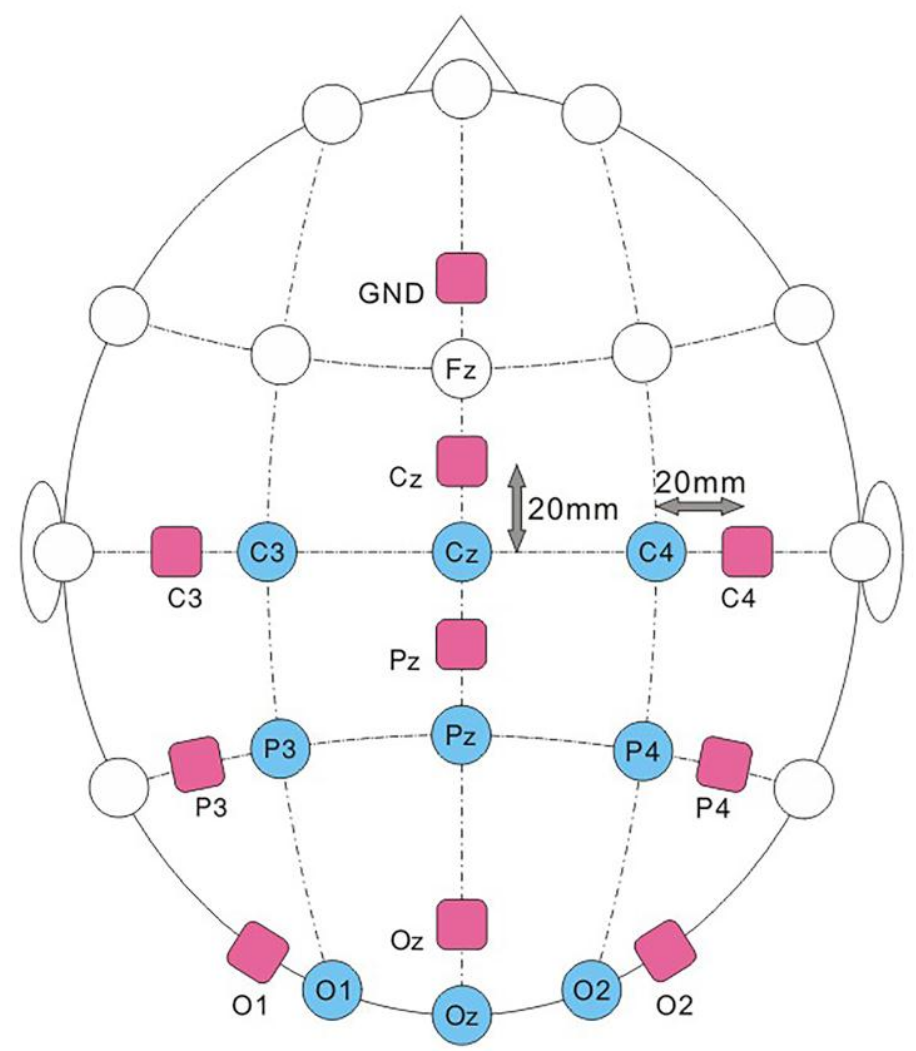

Figure 3 The electrode montage used with both the wet and semi-dry electrodes for in vivo EEG recording, semi-dry electrodes are denoted as blue circles and wet electrodes are denoted as red squares. The reference (not marked) is on the right mastoid. 

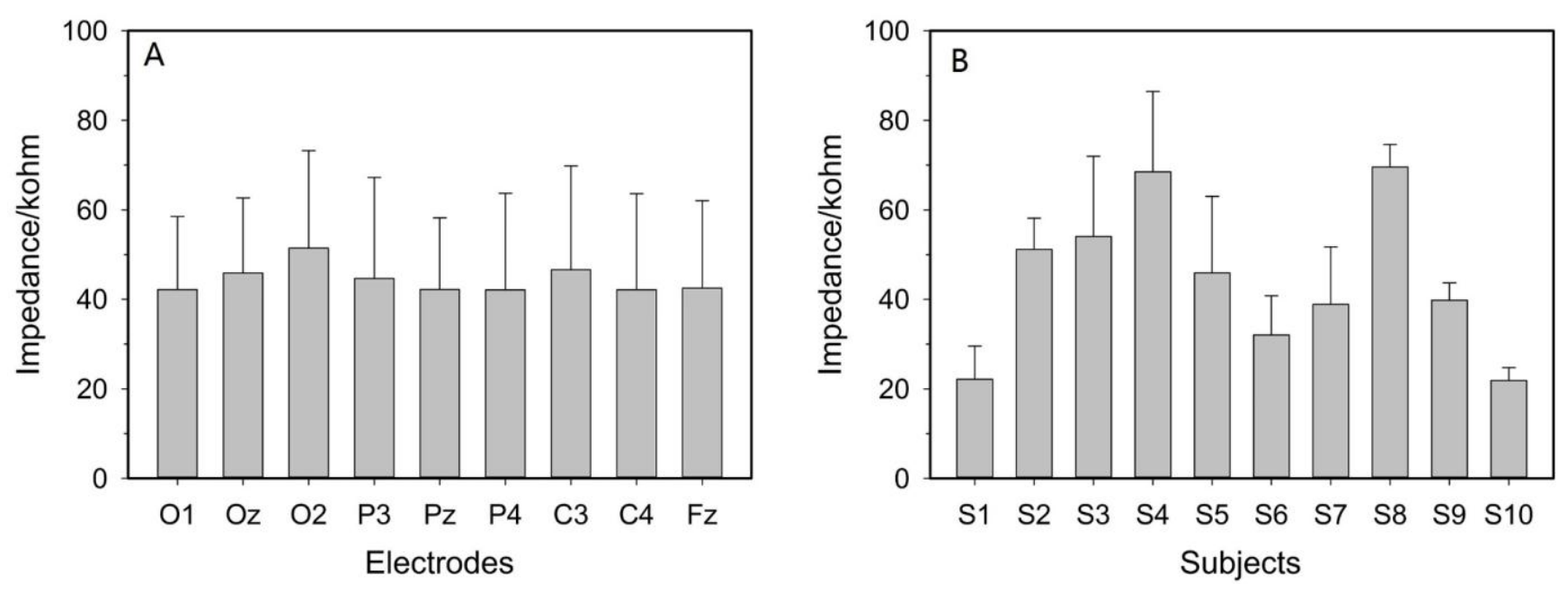

Figure 4 The semi-dry electrode/scalp impedance at $10 \mathrm{~Hz}$ results. (A) A pair of semi-dry electrodes/scalp impedance of nine different positions, tested on ten subjects $(n=10)$. (B) A pair of semi-dry electrodes/scalp impedance of ten different subjects, tested on nine different positions $(\mathrm{n}=9)$; A single electrode impedance can be half of the above value approximately. 
A

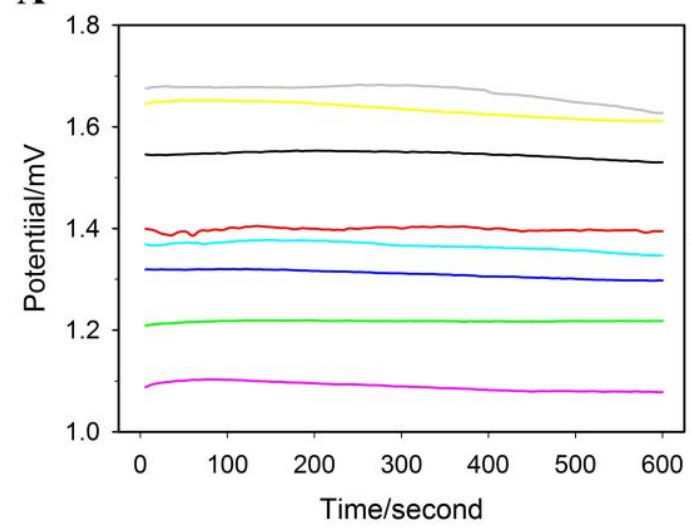

B

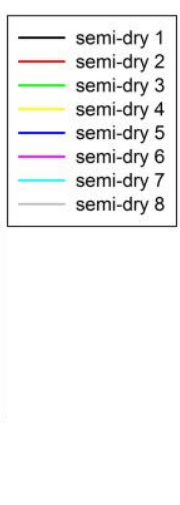

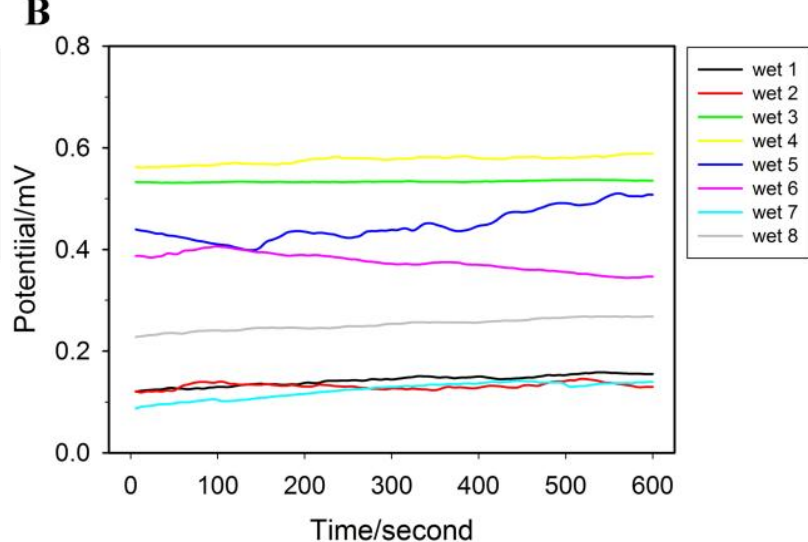

Figure 5 Open circuit potential of eight semi-dry electrodes (A) and $\mathrm{Ag} / \mathrm{AgCl}$ electrodes (B) used in the semi-dry electrode against $\mathrm{Ag} / \mathrm{AgCl}$ reference since the measured electrode immersed in $3.5 \%$ saline solution $10 \min (\mathrm{n}=8)$. 


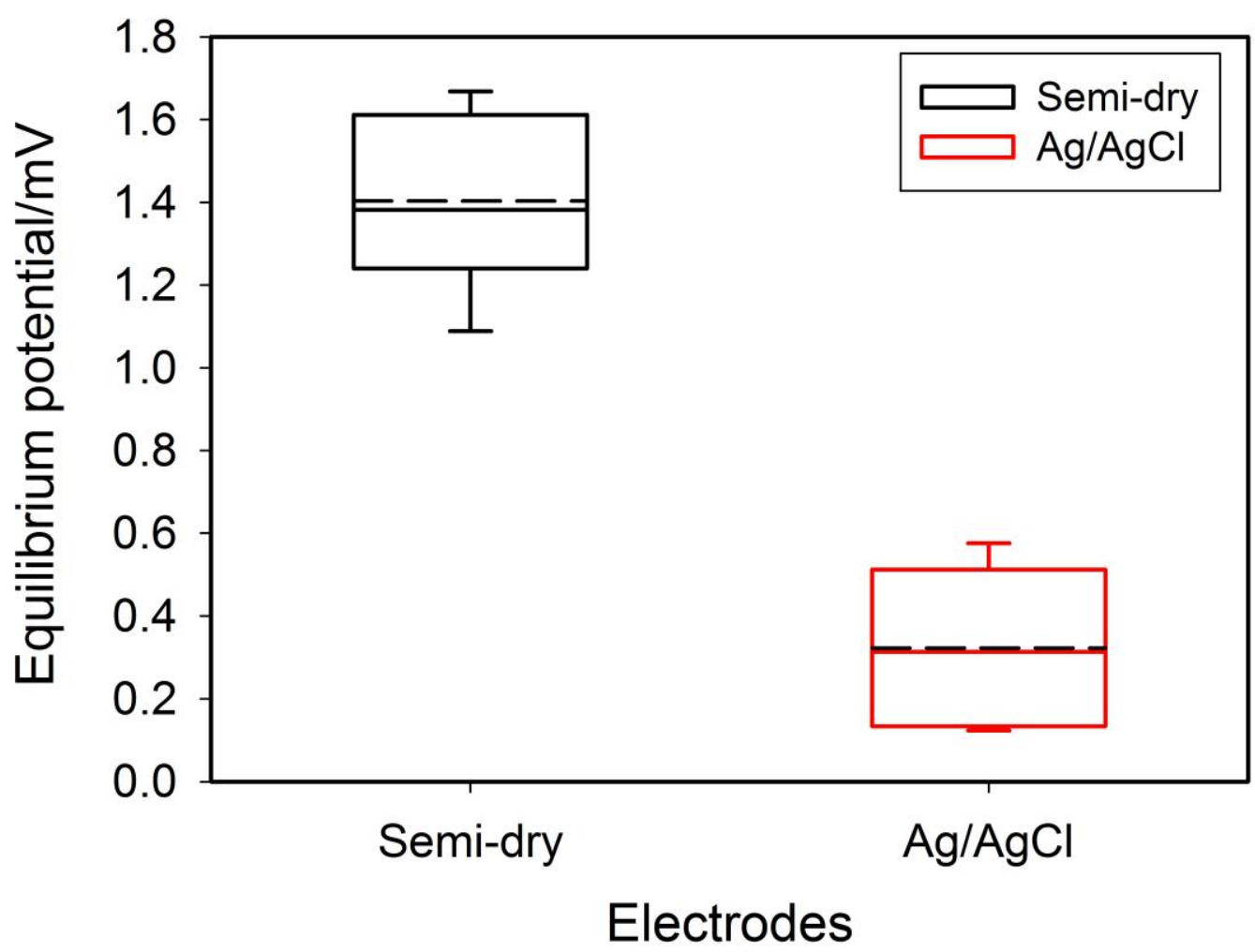

Figure 6 Box-and-whisker plot comparing the equilibrium potential between semi-dry electrodes (left) and $\mathrm{Ag} / \mathrm{AgCl}$ electrodes (right) used in the semi-dry electrode. In the Box-and-whisker plot, the central box represents the value from the lower to upper quartile (25th to 75 th percentile). The middle line represents the median while the dash line represents the mean. The vertical line extends from the minimum to the maximum value, excluding 'outside' and 'far out' values which are displayed as separate points. 

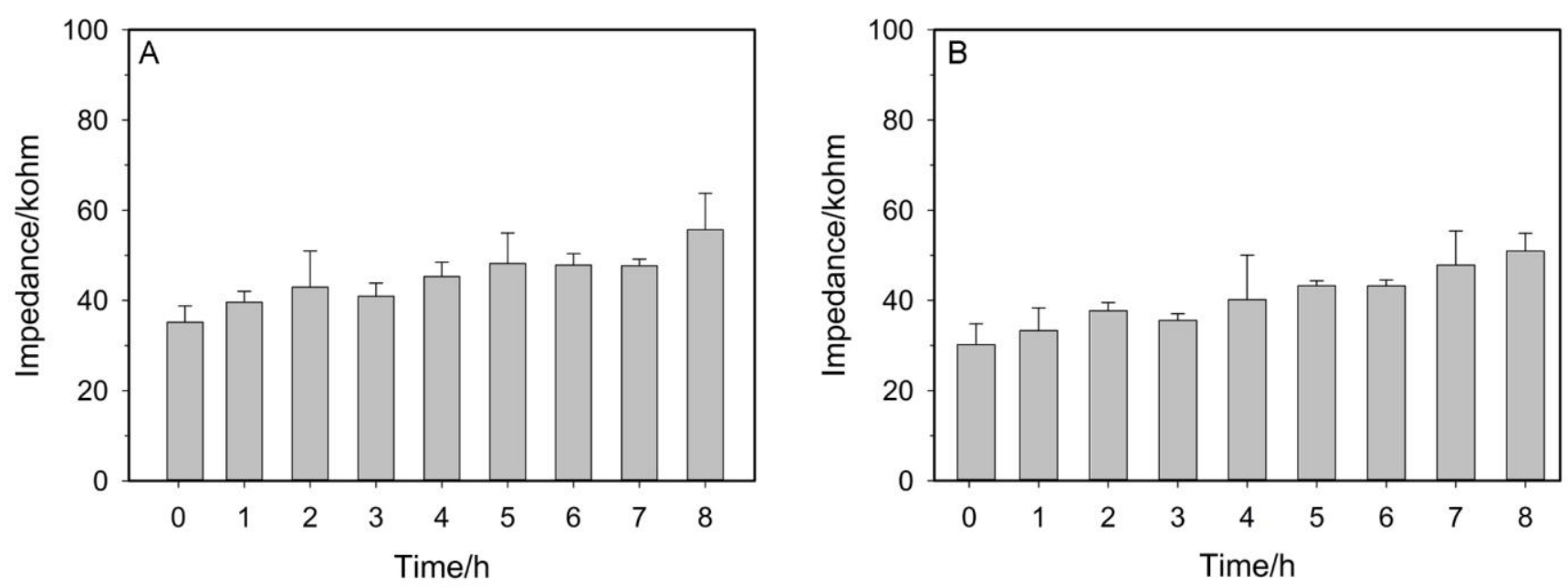

Figure 7 Long-term stability of electrode performance over time by measuring the mean semi-dry/scalp impedance at subject \#6 (A) and subject \#9 (B) for four representative semi-dry electrode pairs within 8 h. 


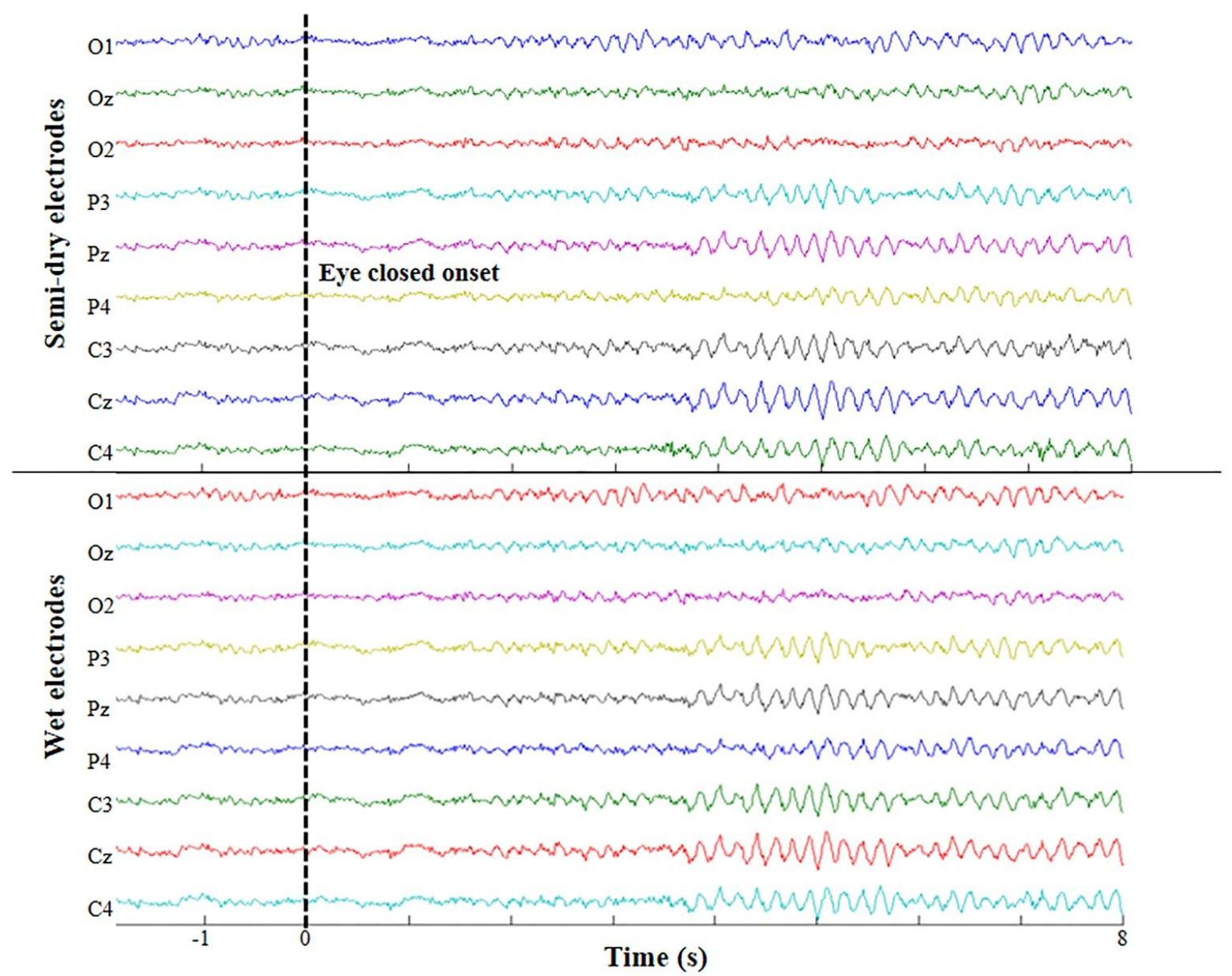

Figure 8 An example of the semi-dry and wet electrodes recordings in the eyes open/closed paradigm for all nine channels in time domain. 

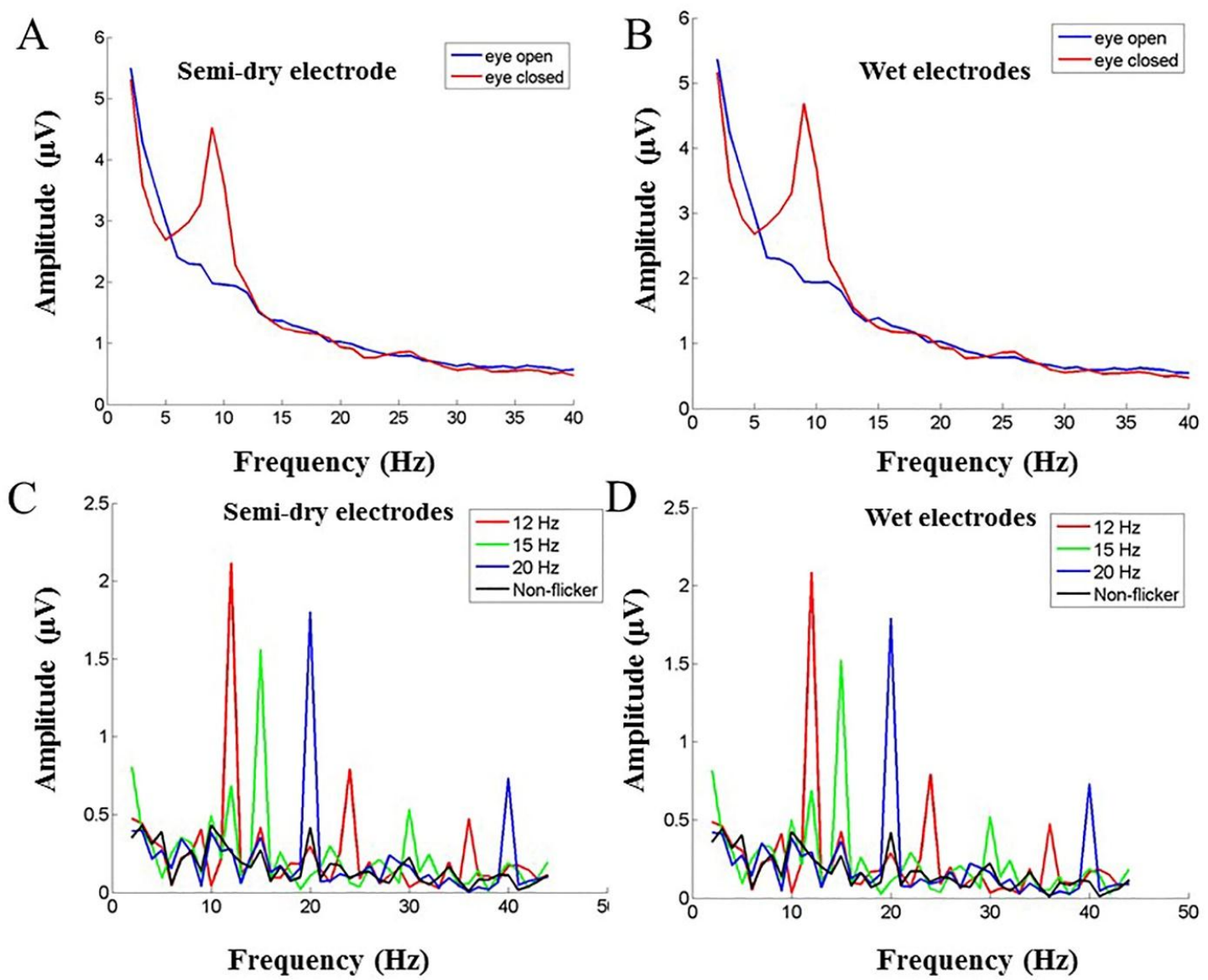

Figure 9 Results of EEG recording in eye open/closed paradigm and SSVEPs paradigms: pectrum of semi-dry (A) and wet (B) electrode Pz from a representative subject (\#5) in eye open/closed paradigm; spectrum of semi-dry (C) and wet (D) electrode Oz from a representative subject (\#4) in SSVEPs paradigm. 

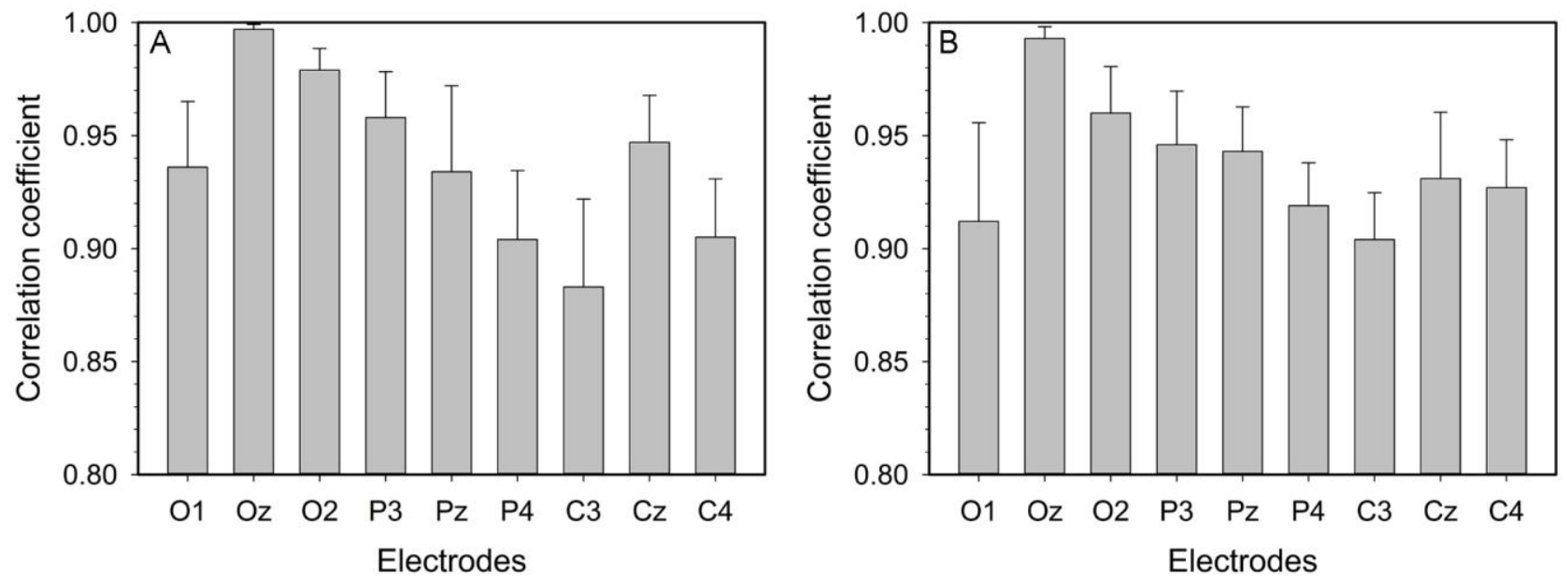

Figure 10 The temporal correlations between the paired wet and semi-dry electrode groups for eyes open/closed paradigms (A) and SSVEP paradigm (B) at the nine recording sites. 
(A)

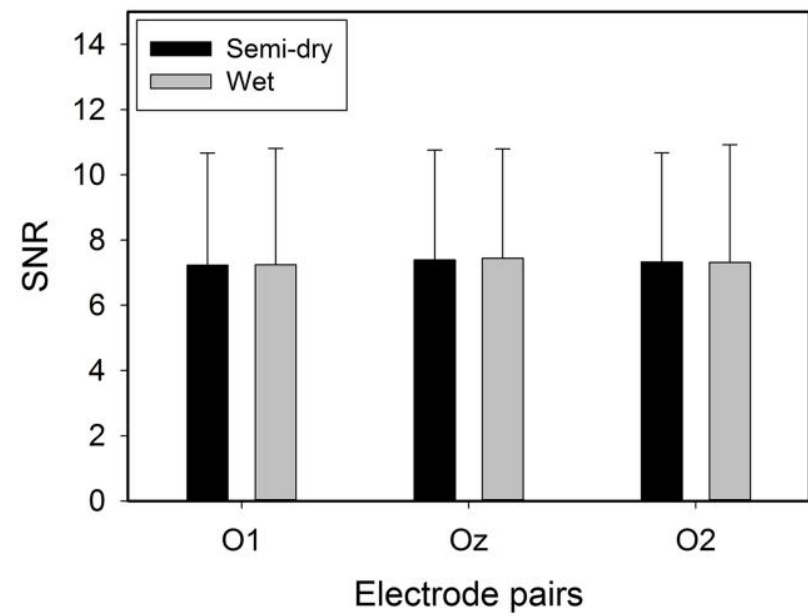

(C)

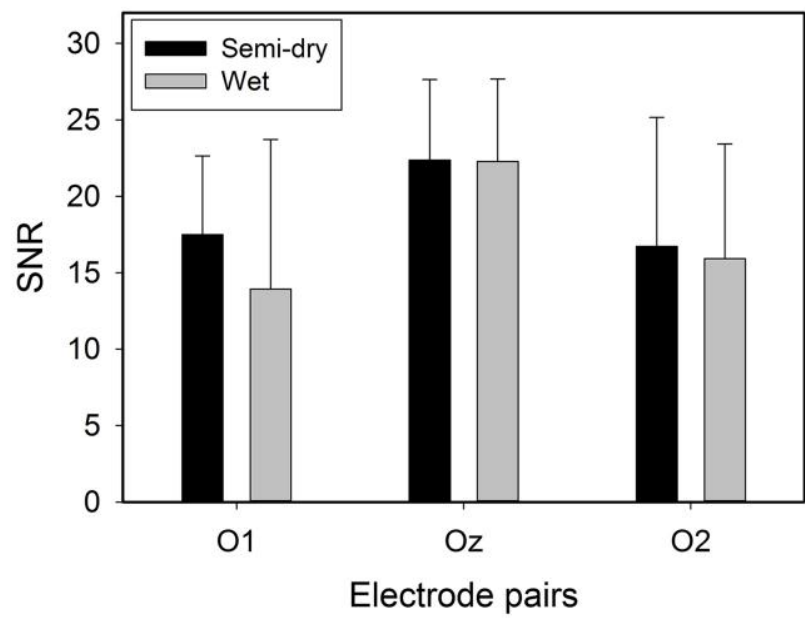

(B)

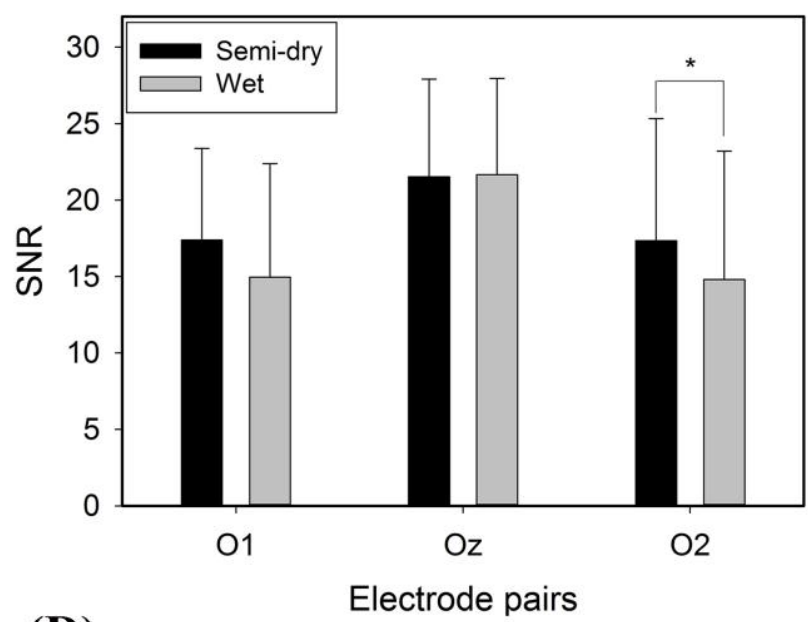

(D)

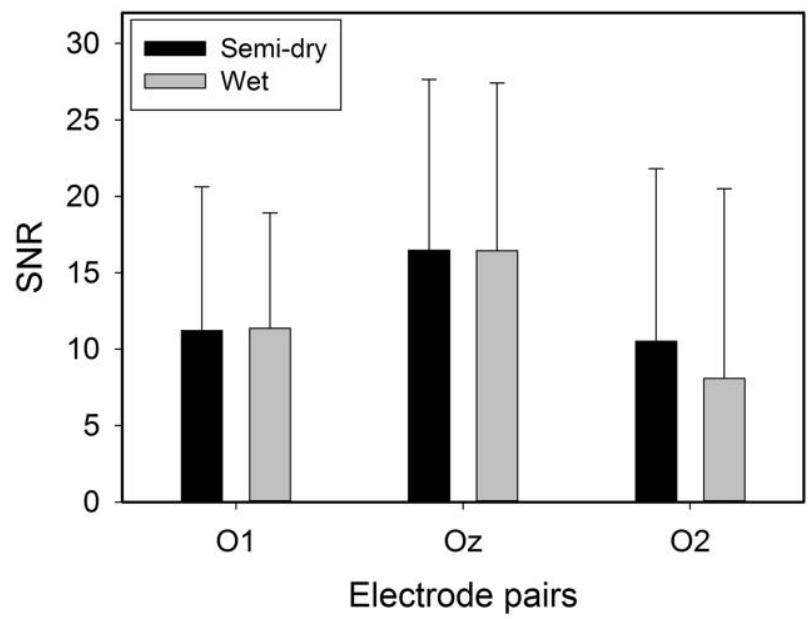

Figure 11 Quantitatively assess the paradigm-specific EEG signal (A: eyes open/closed paradigm; B: $12 \mathrm{~Hz}$ SSVEP paradigm; C: $15 \mathrm{~Hz}$ SSVEP paradigm; D: $20 \mathrm{~Hz}$ SSVEP paradigm) qualities by the SNRs over all ten subjects for occipital domain, and paired t-student was applied for all adjacent electrode pairs $(\mathrm{n}=10, * \mathrm{P}<0.05)$. 